\title{
The review of Trichoplax adhaerens genome: comparative analyses
}

\author{
E. Vodiasova*, E. Chelebieva \\ A.O. Kovalevsky Institute of Marine Biological Research of RAS, Sevastopol, Russia \\ *e-mail: eavodiasova@gmail.com
}

Key words: Trichoplax adhaerens, mitochondrial genome, Placozoa

Motivation and Aim: Trichoplax adhaerens Schulze 1883 is the basal multicellular animal, which structure is very simple: the body consists from three layers, it doesn't have any tissue differentiation, neurons, muscles, synapsis [1]. At that time, this interesting organism is able to change the body form in response to environmental conditions, has different cycles of moving, incomprehensible behavior, the life cycle stages aren't studied completely (for example planktonic forms or sexual reproduction). The Trichoplax has 19 haplotypes with the high genetic divergence. The mitochondrial genome was sequenced in 2006 [2]. The mitochondrial genome is bigger than the other types of animals and has 43079 nucleotides. And two years later the whole genome was sequenced, the size - $98 \mathrm{Mb}, 11514$ genes was found. The presence of different transcription factors (LIM-homeobox and POU- homeobox), genes which involved to syntheses of dopamine, adrenaline and noradrenaline; genes coding different intracellular matrix proteins [3]. But all these genes are characteristic for more complex Animals which have neural systems or intracellular matrix. All of these make the Trichoplax adhaerens as an interesting object for investigation of different evolution process and comparative genome analyses among others animal phyla.

Methods and Algorithms: We analyzed the whole genome (NCBI: GCA_000150275.1) and mitochondrial genome (NCBI: DQ112541.1) of Trichoplax adhaerens, made statistic of presence protein families and comparison with the representatives of different animal phyla.

Conclusion: Despite of simplicity of body structure, the genome of the Trichoplax is very complicated and comparable with other animals. The whole complex of genes, which regulated biological processes absent in the Trichoplax, apparently aren't expressed in the cells. This requires genomic investigation of transcriptome on the cell level in the nearest future.

Acknowledgements: Supported by the Ministry of Education and Science of the Russian Federation grant No. 14.W03.31.001.

\section{References}

1. Smith C.L., Varoqueaux F., Kittelmann M., Azzam R.N., Cooper B., Winters C.A., ... Reese T.S. (2014) Novel cell types, neurosecretory cells, and body plan of the early-diverging metazoan Trichoplax adhaerens. Current Biology. 24(14):1565-1572.

2. Dellaporta S.L., Xu A., Sagasser S., Jakob W., Moreno M.A., Buss L.W., ... Schierwater B. (2006). Mitochondrial genome of Trichoplax adhaerens supports Placozoa as the basal lower metazoan phylum. Proceedings National Academy Sciences. 103(23):8751-8756.

3. Srivastava M., Begovic E., Chapman J., Putnam N.H., Hellsten U., Kawashima T., ... Signorovitch A.Y. (2008) The Trichoplax genome and the nature of placozoans. Nature. 454(7207):955. 